\title{
Effect of AGER on the biological behavior of non-small cell lung cancer H1299 cells
}

\author{
QIONG WANG, WENWEN ZHU, GEQIONG XIAO, MENGYU DING, JIAN CHANG and HUI LIAO \\ Department of Oncology, Affiliated Hospital of Shaoxing University, Shaoxing, Zhejiang 312000, P.R. China
}

Received July 29, 2019; Accepted March 26, 2020

DOI: $10.3892 / \mathrm{mmr} .2020 .11176$

\begin{abstract}
Advanced glycosylation end-product specific receptor (AGER) is a multi-ligand cell surface receptor abnormally expressed in lung cancer, and is a member of the immunoglobulin superfamily. Therefore, this study aimed to explore the effect of AGER on the biological behavior of non-small cell lung cancer (NSCLC) H1299 cell line. A microarray-based gene expression profiling analysis of the GSE27262 dataset from the Gene Expression Omnibus (GEO) database was conducted to identify differentially expressed genes, which were verified using The Cancer Genome Atlas (TCGA) database. The expression of AGER in the normal human lung BEAS-2B cell line and NSCLC H1299 cell line was examined using reverse transcription-quantitative PCR. Lentiviral interference and overexpression vectors of AGER were constructed and transfected into H1299 cells using Lipofectamine ${ }^{\circledR}$. AGER expression and biological properties, including cell viability, apoptosis, migration and invasion abilities, in H1299 cells were investigated using MTT, flow cytometry, wound healing and Transwell assays. AGER was expressed at a low level in NSCLC tissues and H1299 cells $(\mathrm{P}<0.05)$. Compared with control cells, AGER overexpression cells displayed decreased cell viability, proliferation, migration and invasion abilities, and significantly increased levels of apoptosis. Furthermore, AGER overexpression increased the expression of Bax and decreased the expression of $\mathrm{Bcl}-2$ in $\mathrm{H} 1299$ cells $(\mathrm{P}<0.05)$, and AGER knockdown displayed the opposite effects on H1299 cells. Therefore, AGER overexpression decreased the proliferation, invasion and migration abilities of H1299 cells, and increased apoptosis. The present study suggested that AGER might serve as a potential molecular marker for NSCLC.
\end{abstract}

Correspondence to: Dr Hui Liao, Department of Oncology, Affiliated Hospital of Shaoxing University, 999 Zhongxing South Road, Shaoxing, Zhejiang 312000, P.R. China

E-mail: liaohuei0210@sina.com

Key words: advanced glycosylation end-product specific receptor, targeted regulation, non-small cell lung cancer, proliferation

\section{Introduction}

At present, lung cancer is the leading cause of malignant tumor-associated mortality worldwide. Despite improvements in preventative and therapeutic strategies in recent decades, the 5 -year survival rate is still low of only $15-20 \%$ (1). Non-small cell lung cancer (NSCLC) accounts for $85 \%$ of lung cancer cases (2). The survival of patients with NSCLC has significantly improved following the development of chemotherapy and molecular targeted therapy. However, due to the high recurrence and metastasis rate, the long-term survival rate remains poor $(3,4)$. Therefore, it has been hypothesized that further investigation into the anticancer functions of small molecule anticancer compounds may identify novel diagnostic and prognostic markers, thereby improving the survival rate of patients (5-7).

Advanced glycosylation end-product specific receptor (AGER) is a member of the immunoglobulin superfamily of cell surface receptors. AGER protein is a multi-ligand receptor that interacts with a wide range of ligands, including advanced glycosylation end products (AGEs), $\beta$-sheet fibrils, $\mathrm{S} 100$ proteins (S100B, S100P, S100A4, S100A6, S100A8/9 and S100A11-13), high mobility family protein-1 and prion $(7,8)$. AGER expression is associated with diabetic angiopathies and thymic hyperplasia, and functions via the Toll-like receptor 4 and AGE/AGER signaling pathways (9). AGER proteins mediate macrophages under normal conditions (10), whereas the cross-linking reaction between AGER protein and the extracellular matrix is enhanced under pathological conditions, resulting in an increased thickness and permeability of the endangium (11). Substantial evidence has suggested that abnormal AGER expression is closely associated with the immune inflammatory response and tumorigenesis (12). A number of studies have also reported that the expression and mutation rate of AGER are highly increased in esophageal cancer (13), as well as in other types of cancer, including breast, gastric and endometrial cancer (14-16). However, a number of studies have reported that AGER expression is significantly downregulated in lung cancer $(13,17-21)$. In addition, AGER is a highly polymorphic gene with single nucleotide polymorphisms, which may be associated with lung diseases, including chronic obstructive pulmonary disease and acute respiratory distress syndrome (22). Furthermore, high expression of AGER protein is associated with pulmonary inflammation and the deterioration of other lung diseases (23). For example, Caraher et al (24) reported the absence of RAGE 
mitigated acute deleterious effects of particulate matter and may be a biologically plausible mediator of PM-related lung disease. The study of Machahua et al (25) has demonstrated that serum AGE/RAGEs are potential biomarkers of idiopathic pulmonary fibrosis pneumonia. It has also been reported that AGER is closely associated with the low survival rate of patients with lung cancer, based on the analysis of an oncogene microarray and The Cancer Genome Atlas (TCGA) database (26). Therefore, the abnormal expression of AGER in lung cancer tissues and cells indicates that AGER serves an important role in lung cancer, which suggests that AGER may represent as a potential therapeutic target during the development of lung cancer.

The present study aimed to explore the effects of AGER on the biological behavior of the NSCLC.

\section{Materials and methods}

Bioinformatics analysis. The NSCLC microarray dataset GSE27262 (27)was obtained from the Gene Expression Omnibus (GEO) database (www.ncbi.nlm.nih. gov/geo). R language 3.5.3 software (https://cran.r-project. org/bin/windows/base/old/3.5.3/) was used to conduct differential analysis. R package 'pheatmap' was used to create the heatmap of differentially expressed genes (DEGs). The expression level of AGER was validated using TCGA database (ualcan.path.uab.edu/cgi-bin/ualcan-res.pl).

Cell culture and transfection. The human normal lung BEAS-2B cell line, the NSCLC H1299 cell line and the human embryonic kidney 293T cell line were purchased from the Cell Bank of Type Culture Collection of the Chinese Academy of Sciences. BEAS-2B and H1299 cell lines were cultured in RPMI-1640 medium (Sigma-Aldrich; Merck KGaA) supplemented with $5 \%$ fetal bovine serum (FBS, Gibco; Thermo Fisher Scientific, Inc.), 293T cell line were cultured in Dulbecco's Modified Eagle's Medium (DMEM,Gibco, Rockville, MD, USA), supplemented with $10 \%$ fetal bovine serum, $100 \mathrm{U} / \mathrm{ml}$ penicillin, and $100 \mu \mathrm{g} / \mathrm{ml}$ streptomycin. All cells were cultured at $37^{\circ} \mathrm{C}$ with $5 \% \mathrm{CO}_{2}$. At 70-80\% confluence, cells were digested with $0.25 \%$ trypsin for $3 \mathrm{~min}$ at room temperature for passage. Cells in the logarithmic growth phase were selected for subsequent experiments and were divided into four groups: lentivirus (LV)-negative control (NC), LV-AGER, small interfering RNA (siRNA/si)-NC, and si-AGER. AGER cDNA was cloned into the pLenti-C-mGFP vector (OriGene Technologies, Inc.). The pLenti-C-mGFP-AGER plasmid (LV-AGER; Invitrogen; Thermo Fisher Scientific, Inc.) and the corresponding pLenti-C-mGFP-NC (LV-NC; Invitrogen; Thermo Fisher Scientific, Inc.) were used with two packaging vectors pspax 2 (Invitrogen; Thermo Fisher Scientific, Inc.) and pMD2.G (Invitrogen; Thermo Fisher Scientific, Inc.) co-transfected into $293 \mathrm{~T}$ cells (cell density: $1.5 \times 10^{4}$ ) at a final concentration of $50 \mathrm{nM}$ at room temperature for at least 5 min using Lipofectamine ${ }^{\circledR} 2000$ (Invitrogen; Thermo Fisher Scientific, Inc.). Lentiviral particles were harvested and filtered to infect $\mathrm{H} 1299$ cells ( $1 \times 10^{5}$ cells/well), and transfected for $48 \mathrm{~h}$ at room temperature for subsequent experiments.

The AGER siRNA and its negative control sequences were designed using BLOCK-iT ${ }^{\mathrm{TM}}$ RNAi Designer (www. invitrogen.com/rnai): siRNA-NC (5'-TGCCCTACCCTA
GTGTGAT-3'), AGER-siRNA1 (5'-TGCTGATCCTCCCTG AGAT-3') AGER-siRNA2 (5'-GCTGATCCTCCCTGA GATA-3') and AGER-siRNA3 (5'-GCCTTATCCCTAACA GCCA-3'). H1299 cells ( $1 \times 10^{5}$ cells/well) were seeded into a 6-well culture plate and cultured to $60-70 \%$ confluency at room temperature. Subsequently, $8 \mu \mathrm{l}$ siRNA (20 $\mu \mathrm{mol} / \mathrm{l})$ was diluted in $250 \mu \mathrm{l}$ serum-free DMEM and incubated for $5 \mathrm{~min}$ at room temperature. Lipofectamine ${ }^{\circledR} 2000$ reagent (Invitrogen; Thermo Fisher Scientific, Inc.) was diluted in serum-free DMEM and added to the diluted siRNAs for $20 \mathrm{~min}$ at room temperature. siRNA-NC, and AGER-siRNA complexes were added to cells for $48 \mathrm{~h}$ at room temperature. Transfection efficiency was measured using reverse transcription-quantitative PCR (RT-qPCR). Interference efficiency was detected using RT-qPCR.

$R T-q P C R$. Total RNA was extracted from H1299 cells according to $\mathrm{TRIzol}^{\circledR}$ reagent instructions (Invitrogen; Thermo Fisher Scientific, Inc.). RNA concentration was determined via UV spectrophotometry. Total RNA was reversely transcribed into cDNA using the PrimeScript RT kit (Takara Biomedical Technology Co., Ltd.) according to the manufacturer's instructions. Subsequently, qPCR was performed according to the instructions of SYBR Green PCR Kit (Qiagen, Hilden, Germany). The following primer pairs were used for qPCR: AGER forward, 5'-GTGTCCTTC CCAACGGCTC-3' and reverse, 5'-ATTGCCTGGCACCGG AAAA-3'; and $\beta$-actin forward, 5'-GTGGGGCGCCCCAGG CACCA-3' and reverse, 5'-CTCCTTAATGTCACGCACGAT TTC-3'. The following thermocycling conditions were used for qPCR: initial denaturation at $95^{\circ} \mathrm{C}$ for $5 \mathrm{~min}$; followed by 30 cycles of $95^{\circ} \mathrm{C}$ for $40 \mathrm{sec}, 57^{\circ} \mathrm{C}$ for $40 \mathrm{sec}$ and $72^{\circ} \mathrm{C}$ for $40 \mathrm{sec}$, with a final extension at $72^{\circ} \mathrm{C}$ for $10 \mathrm{~min}$. AGER mRNA levels were quantified using the $2^{-\Delta \Delta \mathrm{Ct}}$ method (28) and normalized to the internal reference gene $\beta$-actin. RT-qPCR was performed in triplicate.

Western blotting. Total protein was extracted from the H1299 cells using cold NP40 lysis buffer or RIPA buffer (Beyotime Institute of Biotechnology). The protein was quantified using a bicinchoninic acid assay, and $30 \mu \mathrm{g}$ of total proteins were separated via $12 \%$ SDS-PAGE at $30 \mathrm{~mA}$ for $120 \mathrm{~min}$ and transferred to nitrocellulose membranes. Subsequently, the membranes were blocked with $5 \%$ skim milk powder (dissolved in TBS+0.1\% Tween-20) for $60 \mathrm{~min}$ at room temperature. The membranes were incubated overnight at $4^{\circ} \mathrm{C}$ with primary antibodies targeted against: AGER (cat. no. ab3611; 1:1,000; Abcam), Bax (cat. no. ab32503; 1:1,000; Abcam), Bcl-2 (cat. no. ab32124; 1:500; Abcam) and GAPDH (cat. no. ab181602; 1:2,500; Abcam). Following primary incubation, the membranes were incubated with horseradish peroxidase-conjugated anti-rabbit IgG H\&L secondary antibodies (cat. no. ab6721; 1:2,000; Abcam) at room temperature for $120 \mathrm{~min}$. Proteins were visualized using an ECL luminescent kit (Beijing Solarbio Science \& Technology Co., Ltd.). Western blotting was performed in triplicate and protein expression was quantified using Quantity One 4.6.6 software (Bio-Rad Laboratories, Inc.) with GAPDH as the internal reference.

Cell proliferation assay. Cell proliferation was measured using MTT assay (Sigma-Aldrich; Merck KGaA), according to the manufacturer's instruction. Briefly, NSCLC H1299 cells 

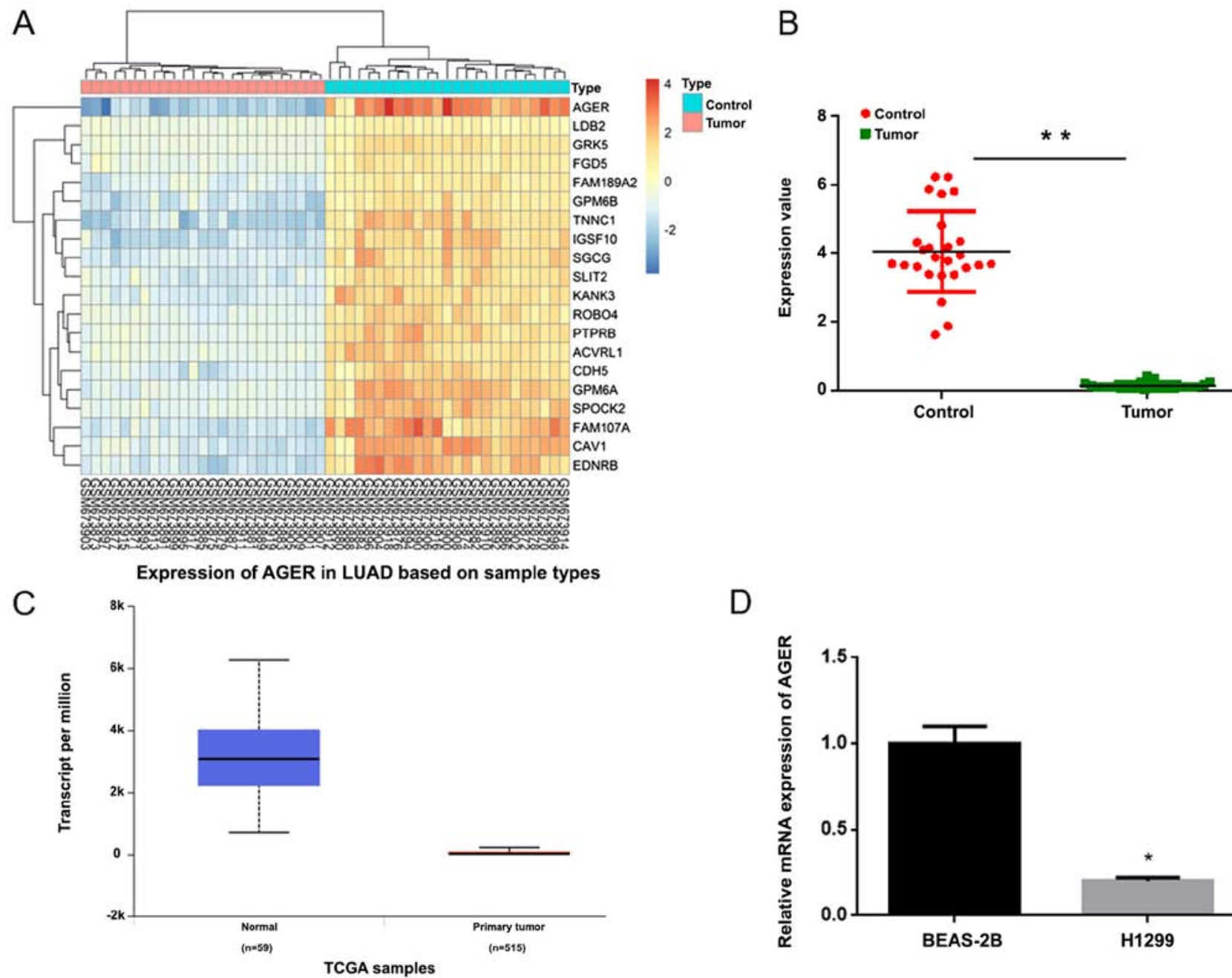

$\mathrm{D}$

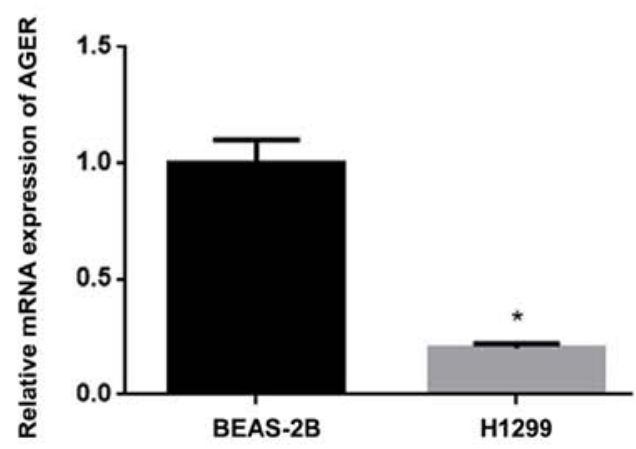

Figure 1. AGER is downregulated in non-small cell lung cancer cells and tissues. (A) DEGs in the GSE12657 dataset were screened. (B) In the GSE12657 data set, AGER exhibited low expression in NSCLC tissue compared with normal tissue. (C) Relative expression of AGER in lung cancer and normal tissues of TCGA database. (D) Relative expression of AGER in the human normal lung BEAS-2B and NSCLC H1299 cell lines, as determined by reverse transcription-quantitative PCR. "P<0.05 and ${ }^{* *} \mathrm{P}<0.01$. AGER, advanced glycosylation end-product specific receptor; DEG, differentially expressed gene; TCGA, The Cancer Genome Atlas.

were seeded in 96-well plates at a density of $2 \times 10^{3}$ cells/well and incubated at $37^{\circ} \mathrm{C}$ for 24,48 and $72 \mathrm{~h}$. Subsequently, $20 \mu \mathrm{l}$ MTT $(5 \mathrm{mg} / \mathrm{ml})$ was added to each well and incubated for $4 \mathrm{~h}$ at $37^{\circ} \mathrm{C}$. Following the MTT incubation, $150 \mu 1$ DMSO was added to dissolve the purple formazan crystals for $15 \mathrm{~min}$ at room temperature. The absorbance of each well at a wavelength of $570 \mathrm{~nm}$ was determined using a microplate reader. The assay was performed in triplicate.

Colony formation assay. H1299 cells were digested with $0.25 \%$ trypsin to individual cells and suspended in culture medium. Cells were seeded at 200 cells per dish and cultured for 3 weeks at $37^{\circ} \mathrm{C}$. When macroscopic clones appeared in the culture dish, cells were fixed with $4 \%$ paraformaldehyde for $15 \mathrm{~min}$ and stained with $0.1 \%$ crystal violet stain for $10 \mathrm{~min}$ at room temperature. The number of colonies ( $\geq 50$ cells/colony) was calculated as follows: Colony formation rate $=($ number of colonies/200) x 100\%. The assay was performed in triplicate.

Flow cytometry. Early and late apoptotic cells were detected using flow cytometry with the Annexin V-FITC Apoptosis
Detection kit (BD Biosciences), according to the manufacturer's protocol. Briefly, H1299 cells were washed twice with cold PBS and resuspended in $200 \mu 1$ PBS. Subsequently, Annexin V-FITC and propidium iodide solution was added to the cells, and the cells were incubated at room temperature for $15 \mathrm{~min}$ in the dark. Finally, flow cytometry (Becton and Dickinson Company) was utilized to detect the apoptotic cells. CELLQuest 3.0 software (Becton and Dickinson Company) was utilized to analyze the data. Flow cytometry was performed in triplicate.

Wound healing assay. A $200 \mu 1$ medium pipette tip was used to scrape a single wound into the H1299 cell monolayer. The monolayer was washed three times with PBS to remove scratched cells. Cells were cultured in DMEM (Thermo Fisher Scientific, Inc.) medium at $37^{\circ} \mathrm{C}$ with $5 \% \mathrm{CO}_{2}$. At 0 and $24 \mathrm{~h}$ time points, five randomly selected fields were observed using an optical inverted microscope (magnification, x100). The migratory rate of the cells was calculated using the following formula: $(0 \mathrm{~h}$ trace width $-24 \mathrm{~h}$ trace width $) / 0 \mathrm{~h}$ trace width. The assay was performed in triplicate. 


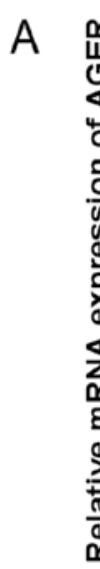

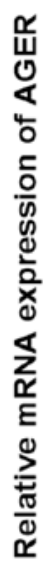

C

\section{LV-NC LV-AGER}

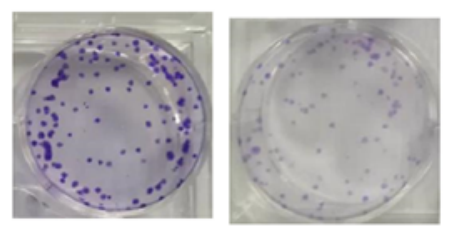

D

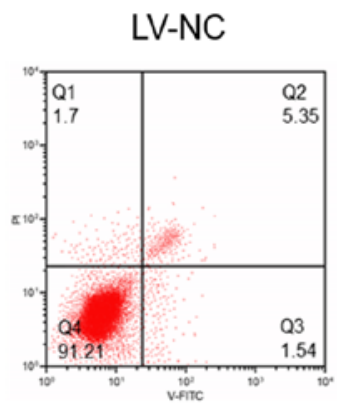

LV-AGER

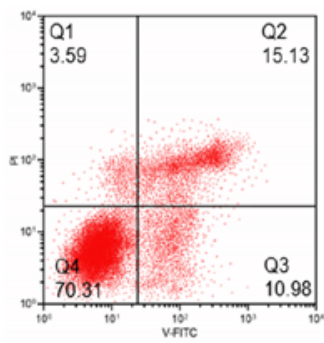

$\mathrm{E}$

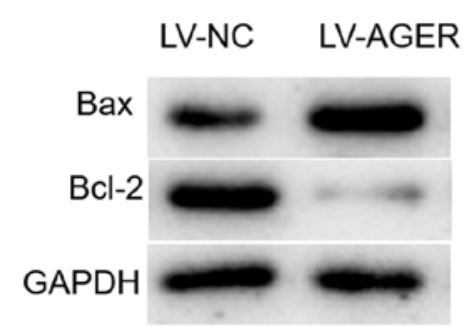

B
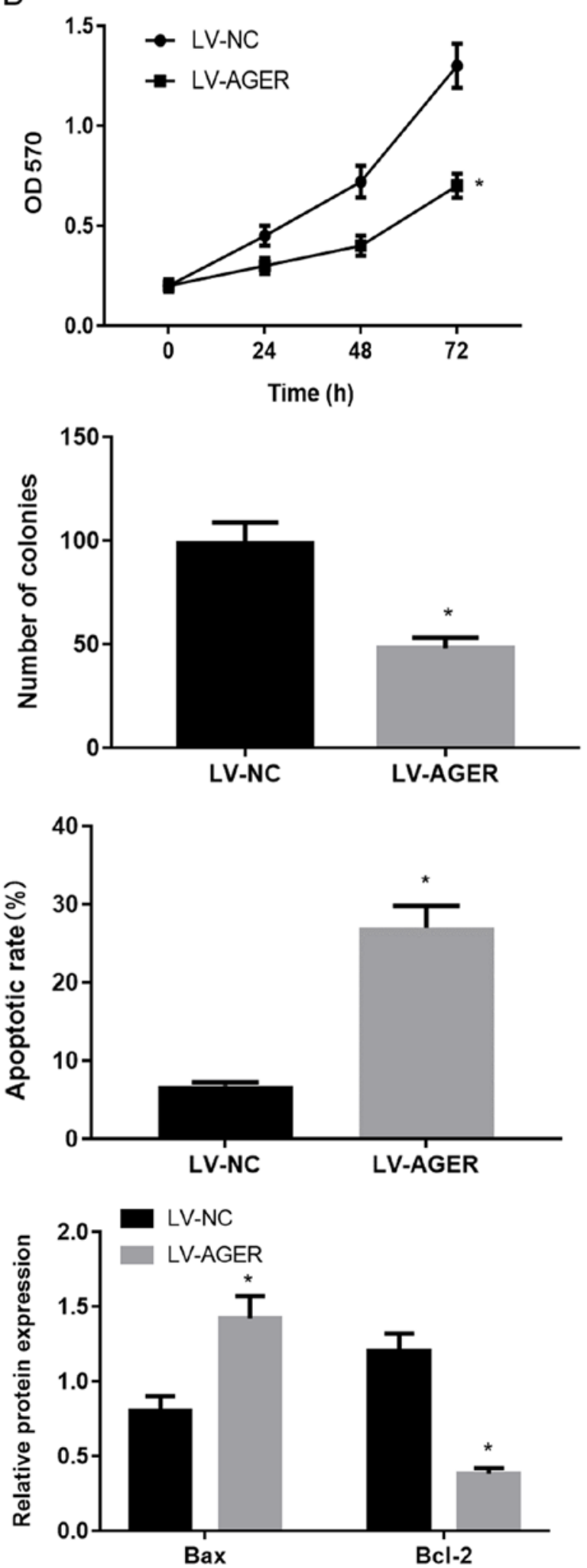

Figure 2. Effects of AGER overexpression on the proliferation and apoptosis of H1299 cells. (A) AGER expression in the LV-NC and LV-AGER groups was determined by reverse transcription-quantitative PCR. (B) Proliferation of the LV-NC and LV-AGER groups was assessed using the MTT assay. (C) Colony formation ability of the LV-NC and LV-AGER groups was determined using the colony formation assay. (D) The apoptotic rate in the LV-NC and LV-AGER groups was detected using flow cytometry. (E) Protein expression levels of Bax and Bcl-2 in the LV-NC and LV-AGER groups were detected using western blotting. *P<0.05 vs. the LV-NC group. AGER, advanced glycosylation end-product specific receptor; LV, lentivirus; NC, negative control; OD, optical density; PI, propidium iodide.

Transwell invasion assay. Each group of H1299 cells was suspended in FBS-free DMEM medium and seeded at a density of $2 \times 10^{5}$ cells/well in the upper chamber of a 24-well
Transwell chamber pretreated with Matrigel for $30 \mathrm{~min}$ at $37^{\circ} \mathrm{C}$. The cells were incubated at $37^{\circ} \mathrm{C}$ for $4 \mathrm{~h}$. DMEM containing $10 \%$ FBS $(500 \mu \mathrm{l})$ was plated in the lower chamber 
A
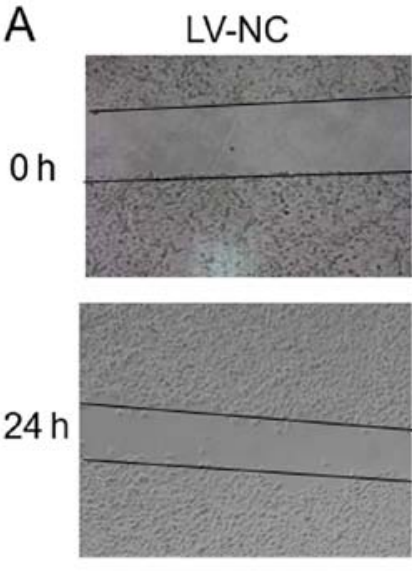

B

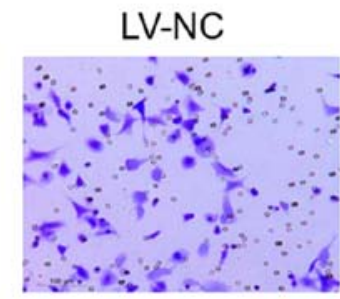

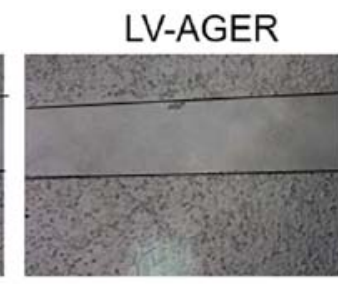
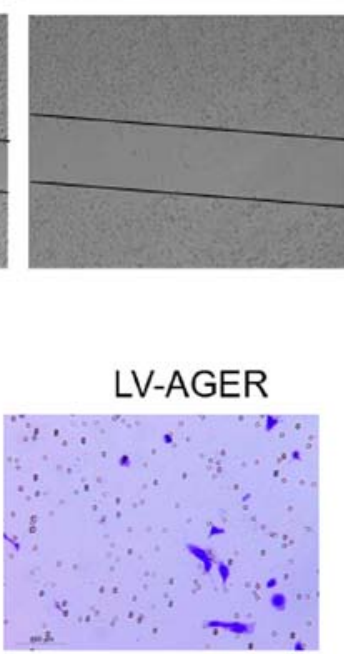
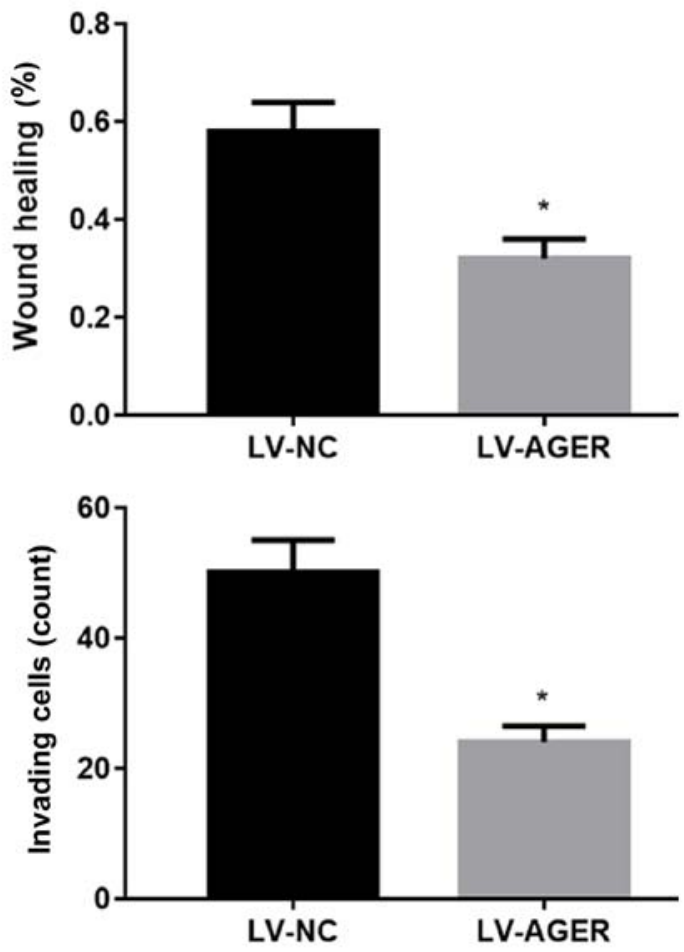

Figure 3. Effects of AGER overexpression on the migration and invasion of H1299 cells. (A) Migratory ability of the LV-NC and LV-AGER groups was determined by the wound healing assay (magnification, x100). (B) Invasive ability of the LV-NC and LV-AGER groups was detected by the Transwell invasion assay (magnification, $\mathrm{x} 100$ ). ${ }^{*} \mathrm{P}<0.05$ vs. the LV-NC group. AGER, advanced glycosylation end-product specific receptor; LV, lentivirus; NC, negative control.

of the Transwell plates. After $24-\mathrm{h}$ incubation at $37^{\circ} \mathrm{C}$, cells on the upper surface of the Transwell membrane were removed using a cotton swab. Invaded cells were fixed with $4 \%$ paraformaldehyde for $20 \mathrm{~min}$ and crystal violet staining for $15 \mathrm{~min}$ at room temperature. Stained cells in six fields of view were observed and counted using an optical inverted microscope at X100 magnification. The assay was performed in triplicate.

Statistical analysis. Statistical analyses were performed using SPSS software (version 21.0; IBM Corp.). Data were presented as the mean \pm standard deviation. Comparisons between two groups were analyzed using paired Student's t-test and multigroup comparisons were made using analysis of variance (ANOVA) with Tukey's post hoc test. $\mathrm{P}<0.05$ was considered to indicate a statistically significant difference.

\section{Results}

AGER expression is downregulated in NSCLC tissues and cells. The microarray GSE27262 dataset was used to analyze DEGs. The results suggested that AGER was the most significant DEG and AGER expression was significantly downregulated in NSCLC tissues compared with control tissues (Fig. 1A and B). AGER was further validated as a DEG using TCGA database (Fig. 1C). Furthermore, RT-qPCR was performed to measure the expression of AGER in the NSCLC cell line. Compared with the normal lung BEAS-2B cell line, AGER expression was significantly decreased in the H1299 cell line (Fig. 1D; $\mathrm{P}<0.05$ ).

AGER overexpression decreases proliferation and promotes apoptosis of H1299 cells. AGER overexpression efficiency in the LV-NC and LV-AGER groups was measured via RT-qPCR. AGER expression in the LV-AGER group was significantly increased compared with the LV-NC group (Fig. 2A; $\mathrm{P}<0.05$ ). To further investigate the effect of AGER on the biological function of H1299 cells, MTT assay was performed to measure H1299 cell viability. The results suggested that proliferation in the LV-AGER group was significantly decreased compared with the LV-NC group (Fig. 2B; P<0.05). Colony formation assays were used to assess alterations in cell clonality, and the results demonstrated that the colony formation ability of the LV-AGER group was decreased compared with the LV-NC group (Fig. 2C; $\mathrm{P}<0.05$ ).

Apoptotic cells were detected by Annexin V-FITC flow cytometry. The apoptotic rate was significantly increased in the LV-AGER group compared with the LV-NC group (Fig. 2D; $\mathrm{P}<0.05$ ). The protein expression of bcl-2 and bax has been confirmed to be closely related to the apoptosis of cancer cells. When the ratio of bcl-2 and bax is down-regulated, it can significantly induce the apoptosis of cancer cells $(29,30)$. The western blotting analysis results indicated that the antiapoptotic protein $\mathrm{Bcl}-2$ was downregulated and the proapoptotic protein Bax was upregulated in the LV-AGER group compared with the LV-NC group (Fig. 2E). The results indicated that AGER overexpression decreased H1299 cell proliferation and promoted apoptosis.

AGER overexpression decreases the migration and invasion of H1299 cells. The migratory ability of H1299 cells was assessed using a wound healing assay, and the results demonstrated that cell migration was significantly decreased in the LV-AGER group compared with that in the LV-NC group (Fig. 3A; $\mathrm{P}<0.05$ ). Furthermore, Transwell invasion assays 
A

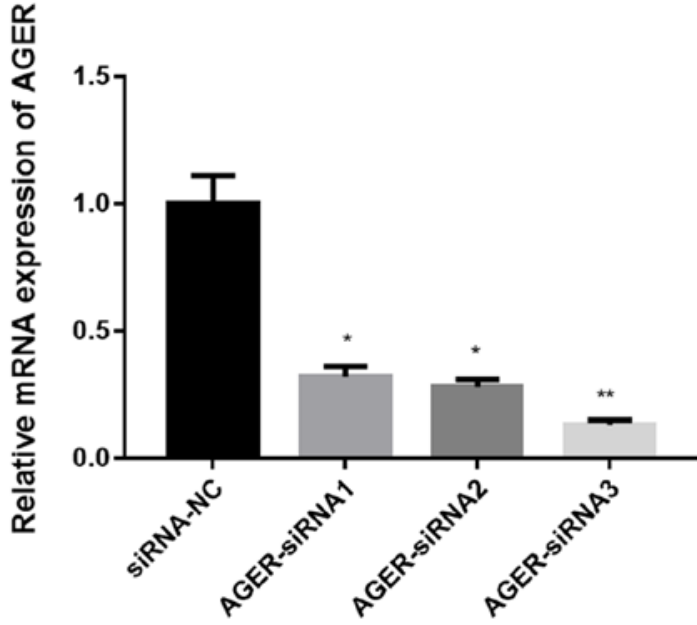

C
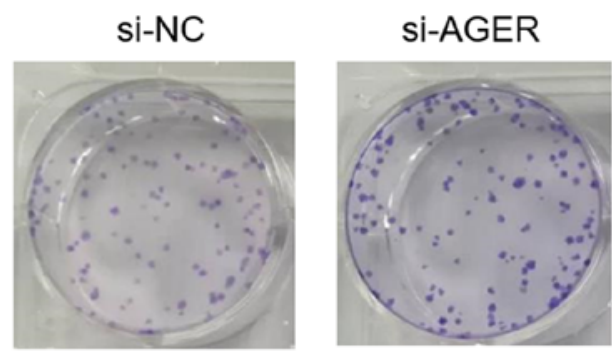

D
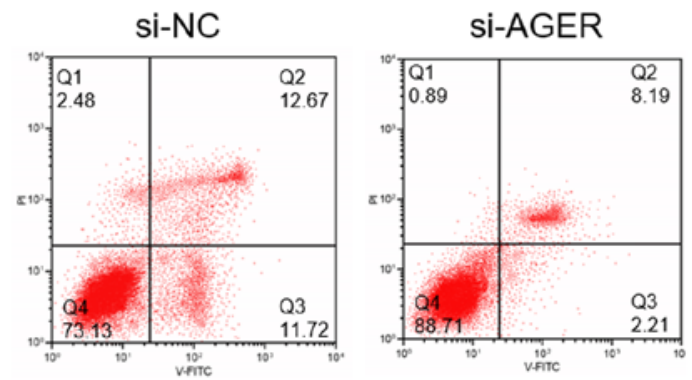

E

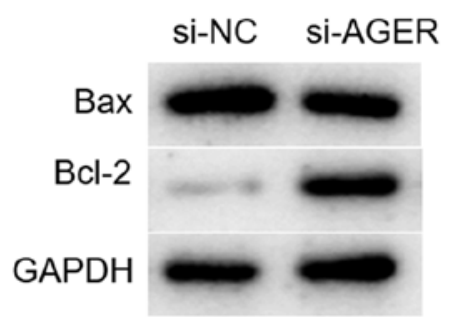

B
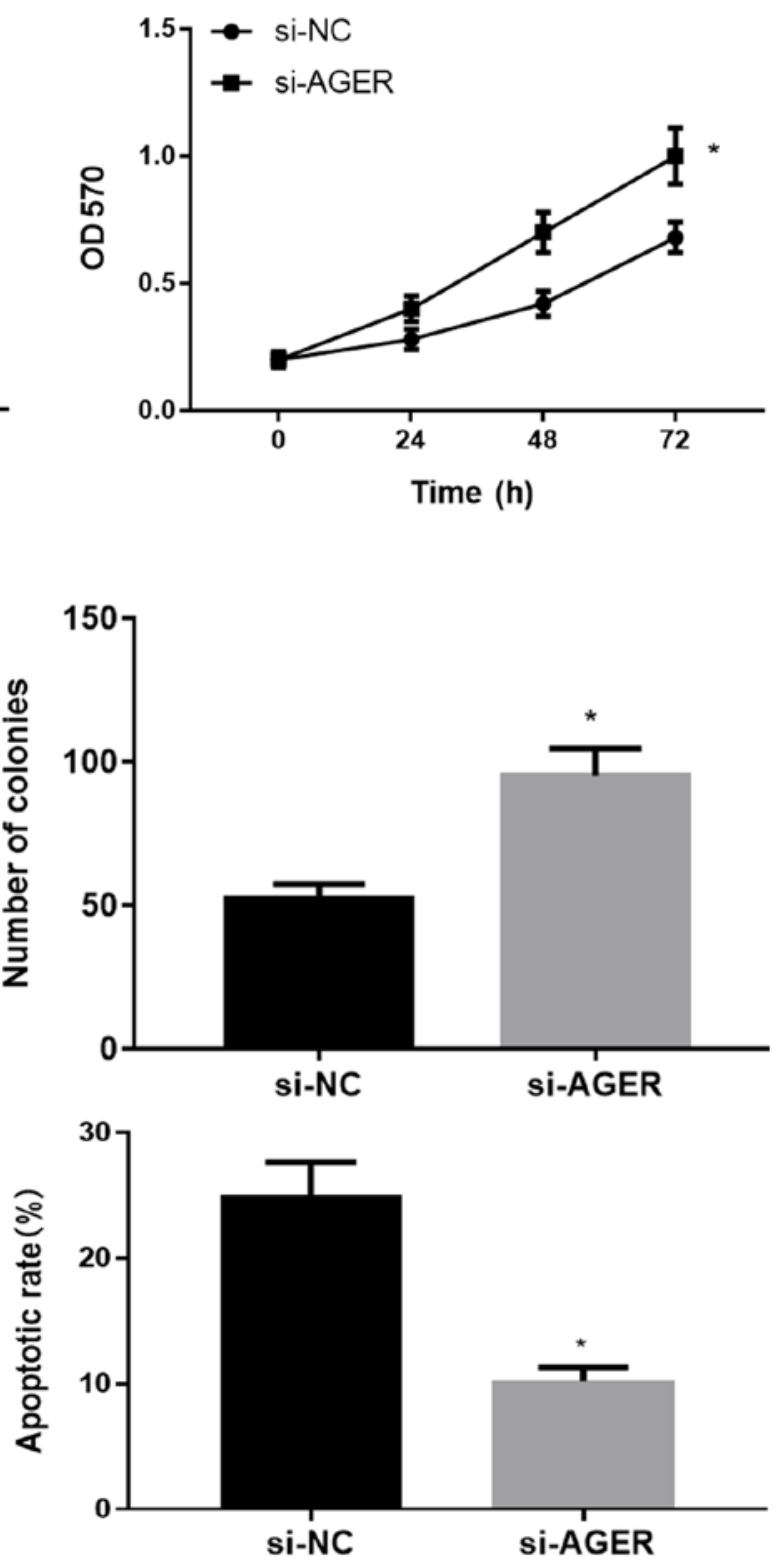

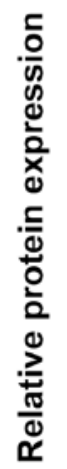

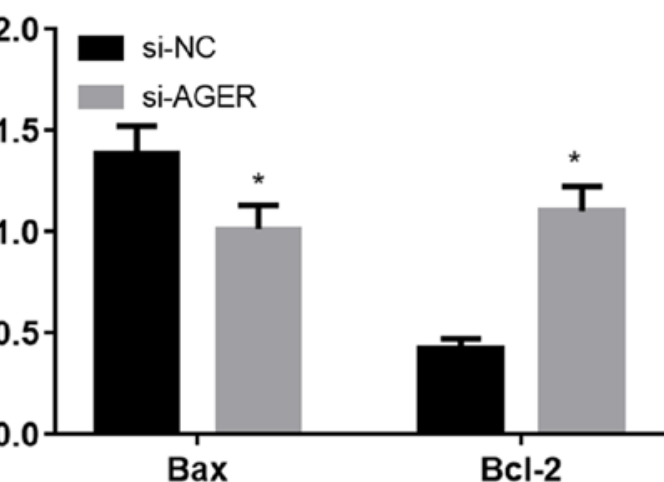

Figure 4. Effects of AGER knockdown on the proliferation and apoptosis of H1299 cells. (A) Transfection efficiency was determined via reverse transcription-quantitative PCR. (B) Proliferation of each group was determined by the MTT assay. (C) Colony formation ability of each group. (D) The apoptotic rate in each group was determined via flow cytometry. (E) Protein expression levels of Bax and Bcl-2 in each group was detected by western blotting. $\mathrm{P}<0.05$ and ${ }^{* * *} \mathrm{P}<0.01$ vs. the si-NC group. AGER, advanced glycosylation end-product specific receptor; si, small interfering RNA; NC, negative control; PI, propidium iodide.

were conducted to investigate the invasive ability of H1299 cells. Compared with the LV-NC group, the invasive ability of the LV-AGER group was significantly decreased (Fig. 3B;
$\mathrm{P}<0.05)$. The results indicated that AGER overexpression decreased the invasion and migration abilities of NSCLC cells. 

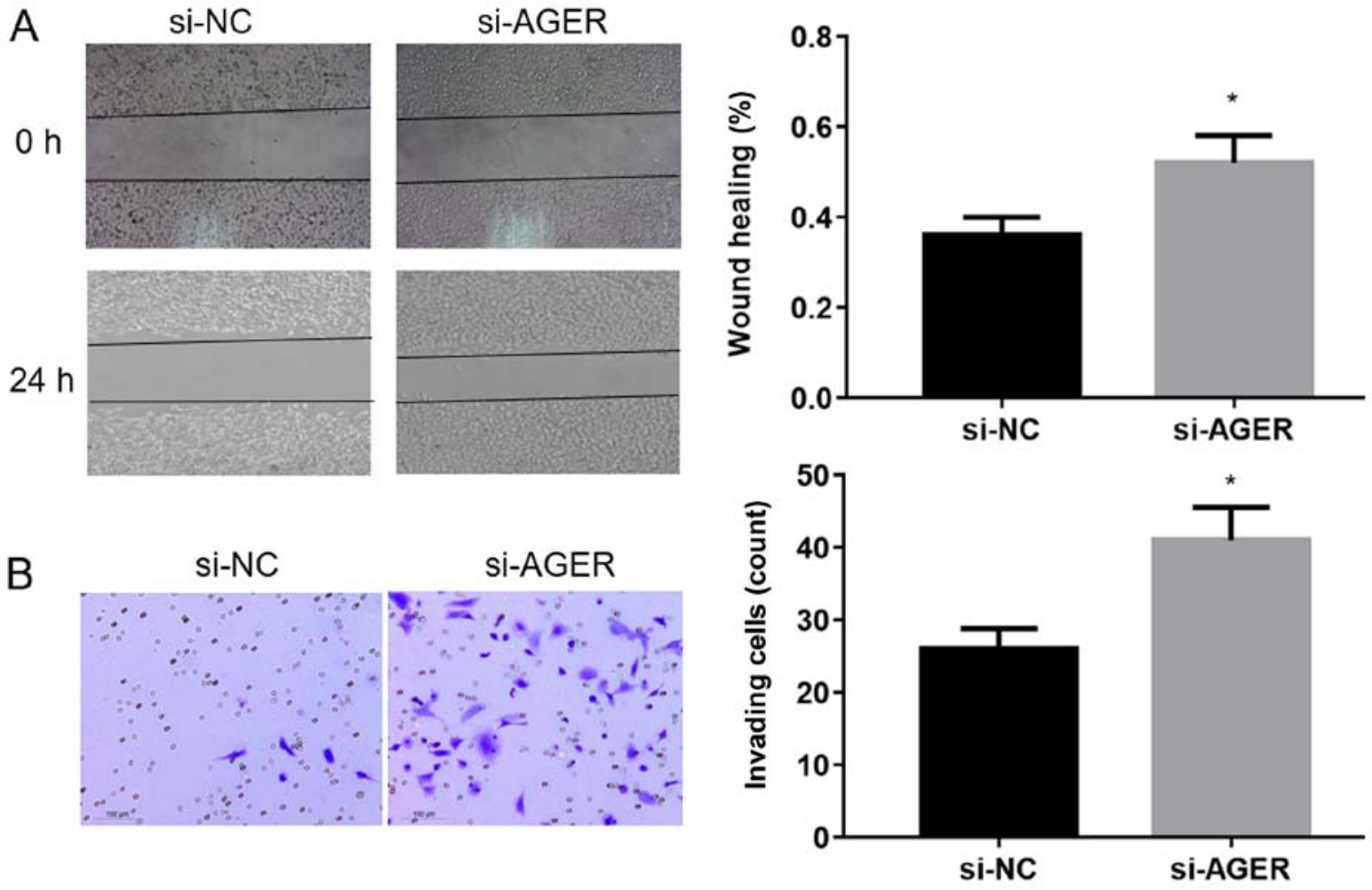

Figure 5. Effects of AGER knockdown on the migration and invasion abilities of H1299 cells. (A) Migratory ability of each group was determined by the wound healing assay (magnification, $\mathrm{x} 100$ ). (B) Invasion ability of each group was detected by the Transwell invasion assay (magnification, $\mathrm{x} 100)$. . $\mathrm{P}<0.05 \mathrm{vs}$. the si-NC group. AGER, advanced glycosylation end-stage specific receptor; si, small interfering RNA; NC, negative control.

AGER knockdown increases proliferation and decreases apoptosis of H1299 cells. The effects of AGER on NSCLC cells were further investigated using AGER knockdown cells. RT-qPCR was performed to measure the transfection efficiency. AGER-siRNA1 and AGER-siRNA2 significantly decreased AGER mRNA expression compared with the siRNA-NC group (Fig. 4A; P<0.05). The most significant reduction in AGER mRNA was observed in the AGER-siRNA3 group compared with the siRNA-NC group (Fig. 4A; $\mathrm{P}<0.01$ ). Therefore, AGER-siRNA3 transfected cells were selected for subsequent experiments.

The results of the MTT assay indicated that cells transfected with AGER-siRNA3 exhibited significantly increased cell viability compared with cells transfected with siRNA-NC (Fig. 4B; $\mathrm{P}<0.05$ ). The colony formation assay results (Fig. 4C) suggested that the colony formation ability of the AGER-siRNA3 group was significantly increased compared with that of the si-NC group $(P<0.05)$. Flow cytometry results indicated that the rate of apoptosis was significantly decreased in the si-AGER group compared with the si-NC group (Fig. 4D; $\mathrm{P}<0.05)$. Western blotting analysis also suggested that $\mathrm{Bax}$ protein expression was significantly downregulated, and Bcl-2 protein expression was significantly upregulated in the si-AGER group compared with the si-NC group (Fig. 4E; $\mathrm{P}<0.05$ ).

The aforementioned results further indicated that AGER knockdown promoted the proliferation and colony formation ability of H1299 cells but decreased apoptosis.

AGER knockdown increases the migration and invasion abilities of H1299 cells. The wound healing assay was performed to assess the migration ability of $\mathrm{H} 1299$ cells transfected with AGER-siRNA3. The results suggested that the migration ability of H1299 cells transfected with AGER-siRNA3 was significantly increased compared with the si-NC group (Fig. 5A; $\mathrm{P}<0.05$ ). The Transwell invasion assay was conducted to investigate the invasive ability of H1299 cells transfected AGER-siRNA3. The AGER-siRNA3 group displayed significantly increased invasive abilities compared with the si-NC group (Fig. 5B; $\mathrm{P}<0.05$ ). The results indicated that AGER knockdown increased the migration and invasion of $\mathrm{H} 1299$ cells.

\section{Discussion}

Analysis of the GEO and TCGA databases indicated that AGER was differentially expressed in NSCLC tissues and downregulated in patients with NSCLC compared with normal controls. Previous studies have also reported that AGER may be used as a potential prognostic biomarker for lung adenocarcinoma $(17,22,31)$, which supports the results of the present study.

To the best of our knowledge, the association between AGER and NSCLC has only been analyzed using bioinformatics analysis in the relevant literature (17,26), and the effects of AGER on the biological behavior of NSCLC have not been reported. Therefore, the effects of AGER on the proliferation, apoptosis and migration of the NSCLC H1299 cell line were investigated in the present study. AGER overexpression reduced the proliferation and colony formation ability of H1299 cells, and increased the rate of apoptosis. AGER overexpression also significantly upregulated Bax protein expression and down- 
regulated Bcl-2 protein expression in $\mathrm{H} 1299$ cells. It has been documented that upregulating Bax or downregulating Bcl-2 promotes apoptosis (30). These results indicated that AGER overexpression decreased the proliferation and increased the apoptosis of H1299 cells.

In addition, the results of the wound healing and Transwell invasion assays indicated that AGER overexpression decreased the migration and invasion of H1299 cells. Transfection experiments were performed to knockdown AGER expression. AGER knockdown increased the proliferation, migration and invasion of H1299 cells and decreased apoptosis. The results further indicated the effects of AGER on the biological behavior of the H1299 cell line. However, the molecular mechanism underlying the effects of AGER on lung cancer requires further investigation. A previous study has reported that AGER may be downregulated in A549 cells due to oxidative-dependent activation of p38 MAPK and NF-kB (26). Another study has also reported that LINC00173 regulates NSCLC via the AGER/NF- $\kappa$ B signaling pathway (18). Therefore, it was hypothesized that AGER may exert its effects on lung cancer via the $N F-\kappa B$ signaling pathway, which requires further investigation.

In present study, the effect of AGER on the proliferation, apoptosis and migration of H1299 cells was investigated. The results further suggested that AGER may serve as a potential therapeutic target for NSCLC. However, there were certain limitations to the present study. For example, the regulatory effect of AGER on NSCLC has not been observed in vivo, and the specific mechanism of AGER action has not been studied in depth. However, the present provided further research evidence for AGER as a potential therapeutic target for NSCLC.

In conclusion, the results of the present study demonstrated that AGER was significantly downregulated in the H1299 cell line compared with the normal lung cell line. Functional tests revealed the antioncogenic characteristics of AGER in NSCLC cells, and AGER overexpression decreased the proliferation and migration, and increased apoptosis of NSCLC cells.

\section{Acknowledgements}

Not applicable.

\section{Funding}

No funding was received.

\section{Availability of data and materials}

The dataset (GSE27262) analyzed during the current study is available in the Gene Expression Omnibus (GEO) database (www.ncbi.nlm.nih.gov/geo). The expression level of AGER was validated using TCGA database (ualcan.path.uab.edu/ cgi-bin/ualcan-res.pl).

\section{Authors' contributions}

HL and QW contributed to the study design. WZ conducted the literature search. GX and MD acquired the data from GEO database and performed data analysis. QW wrote the manuscript. JC revised the manuscript. HL gave the final approval of the version to be submitted. All authors contributed to data analysis, drafting and revising the manuscript and agreed to be accountable for all aspects of the work. All authors read and approved the final manuscript.

\section{Ethics approval and consent to participate}

Not applicable.

\section{Patient consent for publication}

Not applicable.

\section{Competing interests}

The authors declare that they have no competing interests.

\section{References}

1. Zhang F, Yang R, Zhang G, Cheng R, Bai Y, Zhao H, Lu X, Li H, Chen S, Li J, et al: Anticancer function of alpha-solanine in lung adenocarcinoma cells by inducing microRNA-138 expression. Tumour Biol 37: 6437-6446, 2016.

2. Sun B, Liu HF, Ding Y and Li Z: Evaluating the diagnostic and prognostic value of serum miR-770 in non-small cell lung cancer. Eur Rev Med Pharmacol Sci 22: 3061-3066, 2018.

3. Shi H, Bi H, Sun X, Dong H, Jiang Y, Mu H, Li W, Liu G, Gao R and $\mathrm{Su}$ J: Tubeimoside-1 inhibits the proliferation and metastasis by promoting miR-126-5p expression in non-small cell lung cancer cells. Oncol Lett 16: 3126-3134, 2018.

4. Torre LA, Siegel RL and Jemal A: Lung Cancer Statistics. Adv Exp Med Biol 893: 1-19, 2016.

5. Chu K, Gao G, Yang X, Ren S, Li Y, Wu H, Huang Y and Zhou C: MiR-512-5p induces apoptosis and inhibits glycolysis by targeting p21 in non-small cell lung cancer cells. Int J Oncol 48: 577-586, 2016.

6. Wei J, Ma Z, Li Y, Zhao B, Wang D, Jin Y and Jin Y: miR-143 inhibits cell proliferation by targeting autophagy-related $2 \mathrm{~B}$ in non-small cell lung cancer H1299 cells. Mol Med Rep 11: 571-576, 2015.

7. Zhou YL, Xu YJ and Qiao CW: MiR-34c-3p suppresses the proliferation and invasion of non-small cell lung cancer (NSCLC) by inhibiting PAC1/MAPK pathway. Int J Clin Exp Pathol 8: 6312-6322, 2015.

8. Li X, Li M, Chen D, Shi G and Zhao H: PAQR3 inhibits proliferation via suppressing PI3K/AKT signaling pathway in non-small cell lung cancer. Arch Med Sci 14: 1289-1297, 2018.

9. Li Q, Xia S, Yin Y, Guo Y, Chen F and Jin P: miR-5591-5p regulates the effect of ADSCs in repairing diabetic wound via targeting AGEs/AGER/JNK signaling axis. Cell Death Dis 9: 566,2018

10. Xu Y, Toure F, Qu W, Lin L, Song F, Shen X, Rosario R, Garcia J, Schmidt AM and Yan SF: Advanced glycation end product (AGE)-receptor for AGE (RAGE) signaling and up-regulation of Egr-1 in hypoxic macrophages. J Biol Chem 285: 23233-23240, 2010.

11. Chong SF, Lee JH, Zelikin AN and Caruso F: Tuning the permeability of polymer hydrogel capsules: an investigation of cross-linking density, membrane thickness, and cross-linkers. Langmuir 27: 1724-1730, 2011.

12. Bongarzone S, Savickas V, Luzi F and Gee AD: Targeting the Receptor for Advanced Glycation Endproducts (RAGE): A Medicinal Chemistry Perspective. J Med Chem 60: 7213-7232, 2017.

13. Jing RR, Cui M, Sun BL, Yu J and Wang HM: Tissue-specific expression profiling of receptor for advanced glycation end products and its soluble forms in esophageal and lung cancer. Genet Test Mol Biomarkers 14: 355-361, 2010.

14. Nankali M, Karimi J, Goodarzi MT, Saidijam M, Khodadadi I, Razavi AN and Rahimi F: Increased Expression of the Receptor for Advanced Glycation End-Products (RAGE) Is Associated with Advanced Breast Cancer Stage. Oncol Res Treat 39: 622-628, 2016. 
15. Wang D, Li T, Ye G, Shen Z, Hu Y, Mou T, Yu J, Li S, Liu H and $\mathrm{Li} \mathrm{G}$ : Overexpression of the receptor for advanced glycation endproducts (RAGE) is associated with poor prognosis in gastric cancer. PLoS One 10: e0122697, 2015.

16. Zheng L, Li D, Zhou YM, Yang H, Cheng D and Ma XX: Effects of receptor for advanced glycation endproducts on microvessel formation in endometrial cancer. BMC Cancer 16: 93, 2016.

17. Zhang W, Fan J, Chen Q, Lei C, Qiao B and Liu Q: SPP1 and AGER as potential prognostic biomarkers for lung adenocarcinoma. Oncol Lett 15: 7028-7036, 2018.

18. Yang Q, Tang Y, Tang C, Cong H, Wang X, Shen X and Ju S: Diminished LINC00173 expression induced miR-182-5p accumulation promotes cell proliferation, migration and apoptosis inhibition via AGER/NF- $\kappa \mathrm{B}$ pathway in non-small-cell lung cancer. Am J Transl Res 11: 4248-4262, 2019.

19. Fu J, Khaybullin R, Liang X, Morin M, Xia A, Yeh A and Qi X: Discovery of gene regulation pattern in lung cancer by gene expression profiling using human tissues. Genom Data 3 : $112-115,2015$.

20. Pan Z, Liu L, Nie W, Miggin S, Qiu F, Cao Y, Chen J, Yang B, Zhou Y, Lu J and Yang L: Long non-coding RNA AGER-1 functionally upregulates the innate immunity gene AGER and approximates its anti-tumor effect in lung cancer. Mol Carcinog 57: 305-318, 2018.

21. Serveaux-Dancer M, Jabaudon M, Creveaux I, Belville C Blondonnet R, Gross C, Constantin JM, Blanchon L and Sapin V: Pathological implications of receptor for advanced glycation end-product (AGER) gene polymorphism. Dis Markers 2019: 2067353, 2019

22. Liu W, Ouyang S, Zhou Z, Wang M, Wang T, Qi Y, Zhao C, Chen $\mathrm{K}$ and Dai L: Identification of genes associated with cancer progression and prognosis in lung adenocarcinoma: Analyses based on microarray from Oncomine and The Cancer Genome Atlas databases. Mol Genet Genomic Med 7: e00528, 2019.

23. Beucher J, Boelle PY, Busson PF, Muselet-Charlier C, Clement A and Corvol H; French C F Modifier Gene Study Investigators: AGER-429T/C is associated with an increased lung disease severity in cystic fibrosis. PLoS One 7: e41913, 2012.
24. Caraher EJ, Kwon S, Haider SH, Crowley G, Lee A, Ebrahim M, Zhang L, Chen LC, Gordon T, Liu M, et al: Receptor for advanced glycation end-products and World Trade Center particulate induced lung function loss: A case-cohort study and murine model of acute particulate exposure. PLoS One 12: e0184331, 2017.

25. Machahua C, Montes-Worboys A, Planas-Cerezales L, Buendia-Flores R, Molina-Molina M and Vicens-Zygmunt V: Serum AGE/RAGEs as potential biomarker in idiopathic pulmonary fibrosis. Respir Res 19: 215, 2018.

26. de Bittencourt Pasquali MA, Gelain DP, Zeidan-Chulia F, Pires AS, Gasparotto J, Terra SR and Moreira JC: Vitamin A (retinol) downregulates the receptor for advanced glycation endproducts (RAGE) by oxidant-dependent activation of p38 MAPK and NF-kB in human lung cancer A549 cells. Cell Signal 25: 939-954, 2013.

27. Wei TY, Juan CC, Hisa JY, Su LJ, Lee YC, Chou HY, Chen JM, Wu YC, Chiu SC, Hsu CP, et al: Protein arginine methyltransferase 5 is a potential oncoprotein that upregulates G1 cyclins/cyclin-dependent kinases and the phosphoinositide 3-kinase/AKT signaling cascade. Cancer Sci 103: 1640-1650, 2012.

28. Livak KJ and Schmittgen TD: Analysis of relative gene expression data using real-time quantitative PCR and the 2(-Delta Delta C(T)) method. Methods 25: 402-408, 2001.

29. Campbell KJ and Tait SWG: Targeting BCL-2 regulated apoptosis in cancer. Open Biol 8: pii: 180002, 2018.

30. Hassan M, Watari H, AbuAlmaaty A, Ohba Y and Sakuragi N: Apoptosis and molecular targeting therapy in cancer. Biomed Res Int. 2014:150845. 2014

31. Yu DH, Huang JY, Liu XP, Ruan XL, Chen C, Hu WD and Li S: Effects of hub genes on the clinicopathological and prognostic features of lung adenocarcinoma. Oncol Lett 19: 1203-1214, 2020.

This work is licensed under a Creative Commons Attribution-NonCommercial-NoDerivatives 4.0 International (CC BY-NC-ND 4.0) License. 